\title{
Bison Documentation Update, NEAMS Fuels Product Line Review Meeting
}

\author{
Stephanie A Pitts
}

September 2018

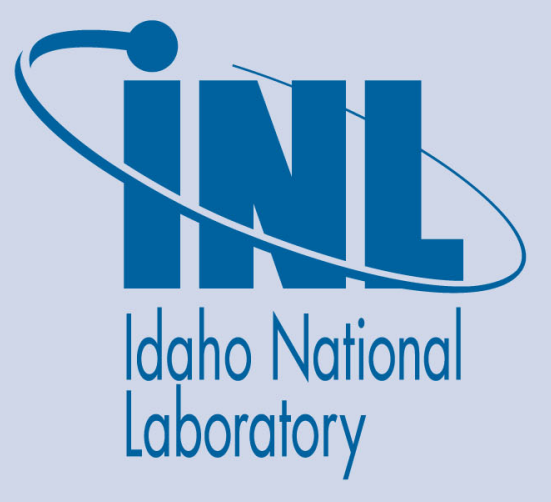

The INL is a U.S. Department of Energy National Laboratory operated by Battelle Energy Alliance 


\title{
Bison Documentation Update, NEAMS Fuels Product Line Review Meeting
}

\author{
Stephanie A Pitts \\ September 2018 \\ Idaho National Laboratory \\ Idaho Falls, Idaho 83415 \\ http://www.inl.gov \\ Prepared for the \\ U.S. Department of Energy \\ Under DOE Idaho Operations Office \\ Contract DE-AC07-05ID14517
}


Stephanie Pitts

NEAMS Fuels Product Line Review Meeting

Idaho Falls, ID

September 19, 2018 


\section{Bison Documentation Objectives}

Nuclear Energy

Flexible, unified, and comprehensive documentation system developed in parallel with source code

- Generated from source code and theory files written in Markdown

- Built with the MooseDocs system

- Merge requests are required to include documentation files

- Recent development Focus on New Users

- Step-by-step setup instructions and tutorials

- Theory and user Unified Documentation implementation

- Detailed unified information for each class and action

- Rapid documentation access for Experienced Users

- Class index pages with integration of relevant MOOSE documentation 


\section{Bison Documentation Objectives}

\section{Nuclear Energy}

\section{Flexible, unified, and comprehensive documentation system}

Bison a Getting Started - Examples \& Tuto

A Finite Element-Based Nuclear Fuel Performance Code

Bison is a finite element-based nuclear fuel performance code applicable to a variety of fuel forms including light water reactor fuel rods, and metallic rod and plate fuel. It is a multiphysics fuel analysis tool that solves fully-coupled thermomechanical problems.

$\pm$

Getting Started

Bison models complex and multiphysics problems integral to Nuclear Fuel Analysis in many different geometries and dimensions, from simplified and fast $1 \mathrm{D}$ simulations to complete and specific $3 \mathrm{D}$ analyses.

Learn how to run Bison on your own machine with our Git Instructions and Example Problem Tutorials.

Bison is built on MOOSE

Bison is based on MOOSE and can efficiently solve problems using standard workstations or very large high-performance computers in a variety of different dimensions, including full 3D, 2D-RZ axisymmetric, layered axisymmetric 1D, and spherically symmetric 1D systems.

\begin{abstract}
.
Code Documentation

Bison fuel models are developed to describe temperature and burnup dependent thermal properties, fission product swelling, densification, thermal and irradiation creep, fracture, and fission gas production and release.

Explore the overview in the Theory Manual and read about the individual code elements in the detailed Code Documentation.
\end{abstract}

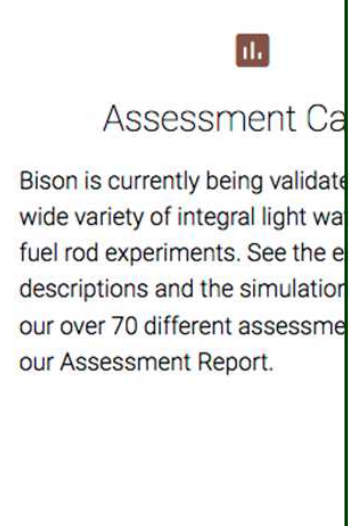

II.

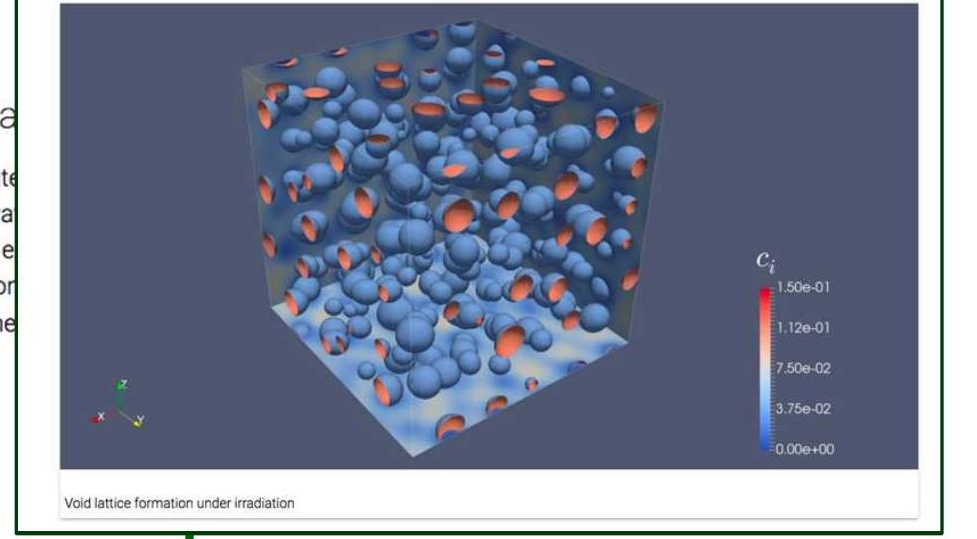

\section{MARMOT}

The NEAMS Mesoscale Fuel Performance Tool class Welcome to the MARMOT project. MARMOT is an Idaho National Laboratory tool focused on modeling the coevolution of microstructure 
Nuclear Energy

\section{Bison Documentation Website}

https://hpcsc.inl.gov/ss|/BISON/site/ (Requires a Bison license, Built daily from Bison's master branch)

https://bison.inl.gov/Documentation/index.aspx (External)

With a bison-opt executable, build the documentation locally

cd $\sim /$ projects/bison/docs

./moosedocs.py build --serve 


\section{Bison Q}

getting_started > gitting_bison

\section{'Git'-ting Bison: Working with Git}

These instructions are meant to help users of both local machines anc installation and build processes are the same for both with the except

\section{NOTE}

These instructions are designed for new users that have never forke

\section{Examples \& Tutorials - \\ Theory $>$ \\ Documentation - \\ Help $>$

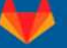

Getting Git Setup

Cloning Bison

Building Bison

Running Bison

Bison Input File

Mesh Script

Contributing
Requesting Access Install the MOOSE Pack... Adding your SSH keys Git Overview Fork Bison Load Modules

Bison and MOOSE use git for code management and distribution. For that reason you will need to learn a little bit about git even if you are not planning on contributing to the code. You will also need access to the Bison repository and a license agreement. Instructions for this are below.

\section{Requesting Access}

Contact a member of the Bison team to get HPC access and a licensing agreement for your institution. This process may take a few weeks. Bison is housed at https://hpcgitlab.inl.gov which lives on the INL HPC system, therefore HPC access is required. Continue on to the next step once HPC access is obtained. 


\section{New User Focus: Examples and Detailed Tutorials}

\section{Examples and Tutorials}

One of the best ways to become familiar with Bison is to start running simulations. To help new users, and returning users looking to brush-up their familiarity, explore Bison, the Bison team provides several examples and tutorials for the different facets of Bison capabilities in projects/bison/examples. functionalities available in Bison

\section{Typical LWR Analysis Geometries}

The geometry and dimension used in a fuel analysis problem represents an optimization between simulation speed and localized detail. Presented below are the three most common geometries used in BISON for LWR fuel rod performance analysis; click on the heading to go to the geometry specific tutorial pages.

Use existing example input files

- Represent common simulation options

Explain interaction among common input file blocks

- Highlight parameter settings

\section{Layered 10}

Provides a quick simulation of a simplified fuel analysis model. Layered $1 \mathrm{D}$ models are ideal for simulations in which runtime speed is a prime consideration and localized effects can be neglected.

\section{$\equiv$ \\ 2D Axisymmetric}

The yeoman of Bison fuel analysis simulations. 2D Axisymmetric (2D-RZ) simulations are best suited for modeling the axial and radial variations common in fuel while retaining the computational efficiency of a $2 \mathrm{D}$ problem.

\section{Complete 3D}

Enables the study of localized effects on the performance of a fuel rod. Full 3D models are most beneficial for simulations of non-symmetric fuel rods, including manufacturing defects on a single fuel pellet surface or localized loading conditions.

The most suitable geometry to use in a fuel rod analysis depends on the details of the specific analysis requirements.

\section{Additional Tutorials}

Beyond the light water reactor examples given above, Bison includes tutorials and guidelines for advanced topics, including changing geometry, different fuel rod types, and updating input files to the lastest mechanics capabilities. Click on the headers below to go to the tutorial sections.

$$
\text { ไาำ }
$$

Switching Dimensions

Bison's ability easily upscale and downscale among simulation dimensions
$\Phi$

Advanced Fuel Examples

Bison includes the capability to model many other fuels beyond tradiational LWR

\section{玷}

Tensor Mechanics Migration

The guidelines presented in this section will assist in the process of converting 


\section{New User Focus: Examples and Detailed Tutorials}

\section{Nuclear Energy}

\section{Introduction to Layered 1D Models}

The term 'Layered1D' in Bison describes a model of cylindrical fuel geometry that is a collection of coupled 1D simulations used to represent the behavior of the fuel at given axial positions along the rod. The fuel rod is divided into several axial slices, and a 1D model of the physics, Eq. 1 and Eq. 2, is solved on each slice. An illustration of these $1 \mathrm{D}$ axial slices is shown in Figure 1.

The following equations for energy conservation and stress divergence, respectively, are solved simultaneously using 1D axisymmetric models for each axial slice:

$$
\begin{gathered}
\rho C_{p} \frac{\partial T}{\partial t}-\nabla \cdot k \nabla T-q=0 \\
\nabla \cdot \boldsymbol{\sigma}=0
\end{gathered}
$$

where $\rho$ is the mass density, $C_{p}$ is the specific heat, $T$ is the temperature, $k$ is the thermal conductivity, $q$ is the volumetric heat generation rate, and $\sigma$ is the stress.

Each slice represents the behavior of a finite axial segment of the rod. The behavior is assumed to be uniform over the entirety each axial segement

\section{Layered 1D Specific Code}

Because a given 1D model is only applicable for a finite length of the fuel rod, and generally the behavior of a rod varies significantly over its length, the ability to represent axial variations is necessary. This ability is added to Bison with a set of code classes that compute integral quantities specifically for Layered 1D models, including code used to calculate:

Figure 1: Schematic of Layered 1D Coupled Problem and Mesh

gy conservation and stress divergence are

elements in

contact. Axial effects are accounted for by

summing displacements in each slice using a generalized plane strain formulation.
Introduction to Layere.. Layered 1 D Specific Co... Coupling with Axial Out ... Comparison with $2 \mathrm{D}$ - ... Best Use Cases for Lay... References

- Plenum volume (InternalVolumeLayered1D)

- Fission gas released (SideAverageValueLayered1D computes the average temperature used to determine fission gas released)

- Heat flux (SideFluxIntegralLayered1D)

- Burnup profiles (Layered1DFuelPinGeometry provides geometry information about the fuel rod geometry)

- Axial pressure contributions from the coolant and plenum pressure (out_of_plane_pressure parameter in Layered1DMaster)
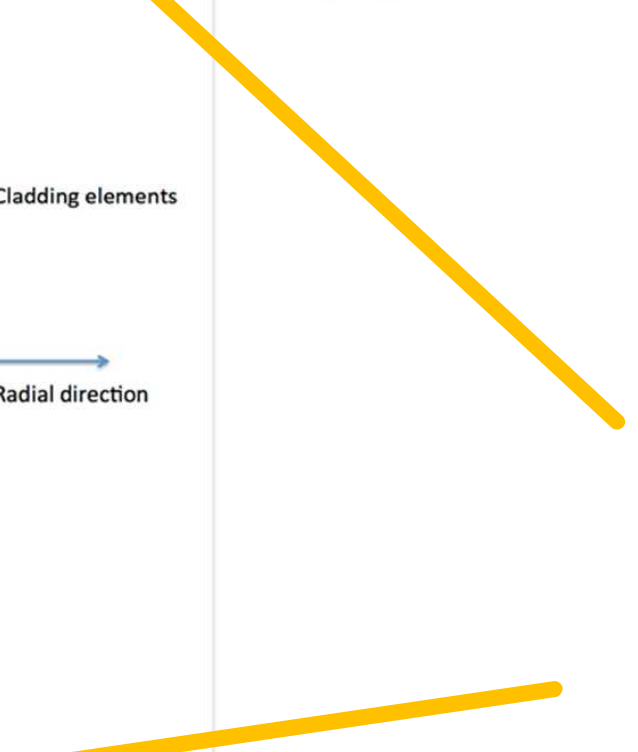


\section{Unified Documentation: \\ Example Class Page}

Nuclear Energy

\section{Internal Volume Layered1D}

Computes the volume of an enclosed area by performing an integral over a user-supplied boundary.

\section{Description}

InternalVolumeLayered1D calculates the internal volume for the case of Layered1D. For the integration along the layer stacking direction, it uses the constant slice_height for each layer fetched from

Layered1DFuelPinGeometry object.

$$
V=\sum_{i} h_{i} \cdot A_{i}
$$

where $V$ is the total integrated volume, $i$ is the layer index, $h_{i}$ is the height of layer $i$, and $A_{i}$ is the in-plane area of layer $i$.

Example Input Syntax

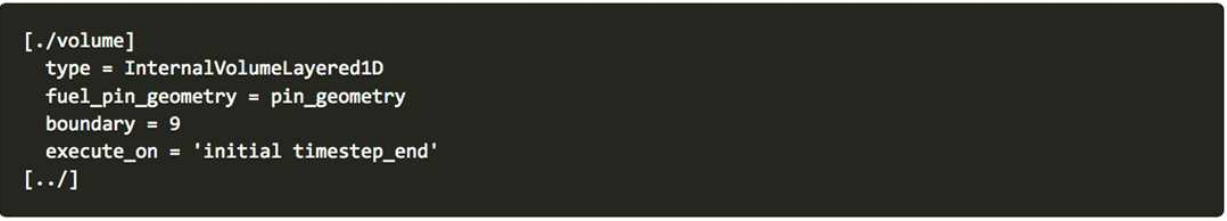

(test/tests/layered_1D/internal_volume.i)

Input Parameters

Required Parameters

out_of_plane_strain The out-of-plane strain nodal variable

boundary The list of boundary IDs from the mesh where this boundary condition applies

fuel_pin_geometry Name of Layered1DFuelPinGeometry UserObject
- Single complete documentation page:

- Theoretical information

- Input file example syntax

- Available input parameters

- Parameters, descriptions, and defaults taken directly from source code

Example input syntax block linked from regression tests 


\section{Unified Documentation: \\ Example Action Page}

Nuclear Energy

\section{Connects action parameters to partial differential equation terms}

\section{Burnup Action System}

Computes the radial distributions of power density, burnup, and concentrations of various heavy metal isotopes in UO2 and U3Si2 fuels for LWRs

\section{Constructed Objects}

Table 1: Correspondence Among Action Functionality and Moose/Bison Objects for the Burnup Action

\section{Functionality}

Calculate radial distributions of power density, burnup,

and heavy metal concentrations

Retrieve and store the radial profiles of burnup and

fisson rate

\section{Replaced Classes}

BurnupFunction
BurnupGrid and AuxVariables
BurnupGrid and AuxVariables

\section{Associated Parameters}

fuel_inner_radius and fuel_outer_radius: values of the fuel radial dimensions

a_lower and a_upper: values of the fuel axial dimensions num_radial: integer of the number of burnup-specific grid divisions in the radial direction

num_axial : integer of the number of burnup-specific grid divisions in the axial direction

rod_ave_lin_pow: a string containing the name of the average rod linear power function

burnup: string containing the name of the burnup auxvariable (created by default)

fisson_rate: string cof the name of the fission rate auxvariable (created by default)

average_burnup: string containing the name of the average burnup auxvariable

N235, N236, N238, N240, N241, N242: string containing the name of the heavy metal ion of interest
Description

Constructed Objects Example Input Syntax Input Parameters Associated Actions 


\section{Bison Only Code Reference Manual}

Given below are the reference pages for all of the Bison specific code. To see a complete list of all of the possible input parameter options and the associated reference pages for Bison and the MOOSE Modules code, view the Complete Code Manual.

Click the blue links in the class names shown below to view the detailed description the class purpose, theoretical models, input file examples, and references.

\section{AuxKernels $\rightarrow$}

Al203Aux Calculates the Aluminum oxide th

BurnupAux Compute burnup given fission_ra

BurnupGrid Retrieve burnup, fission_rate, con

BurnupMetalAux

CoolantAux

CumulativeDamagelndex Calculates the cur

EffectiveBurnupAux Compute effective burn

EutecticThicknessFCCI Calculates eutectic p

FastNeutronFluenceAux Compute fast neut

FastNeutronFluxAux Compute fast neutron

\section{| AuxKernels}

AuxVariables

BCs

Burnup

CladdingHydrides

CoolantChannel

DefaultElementQuality

Functions

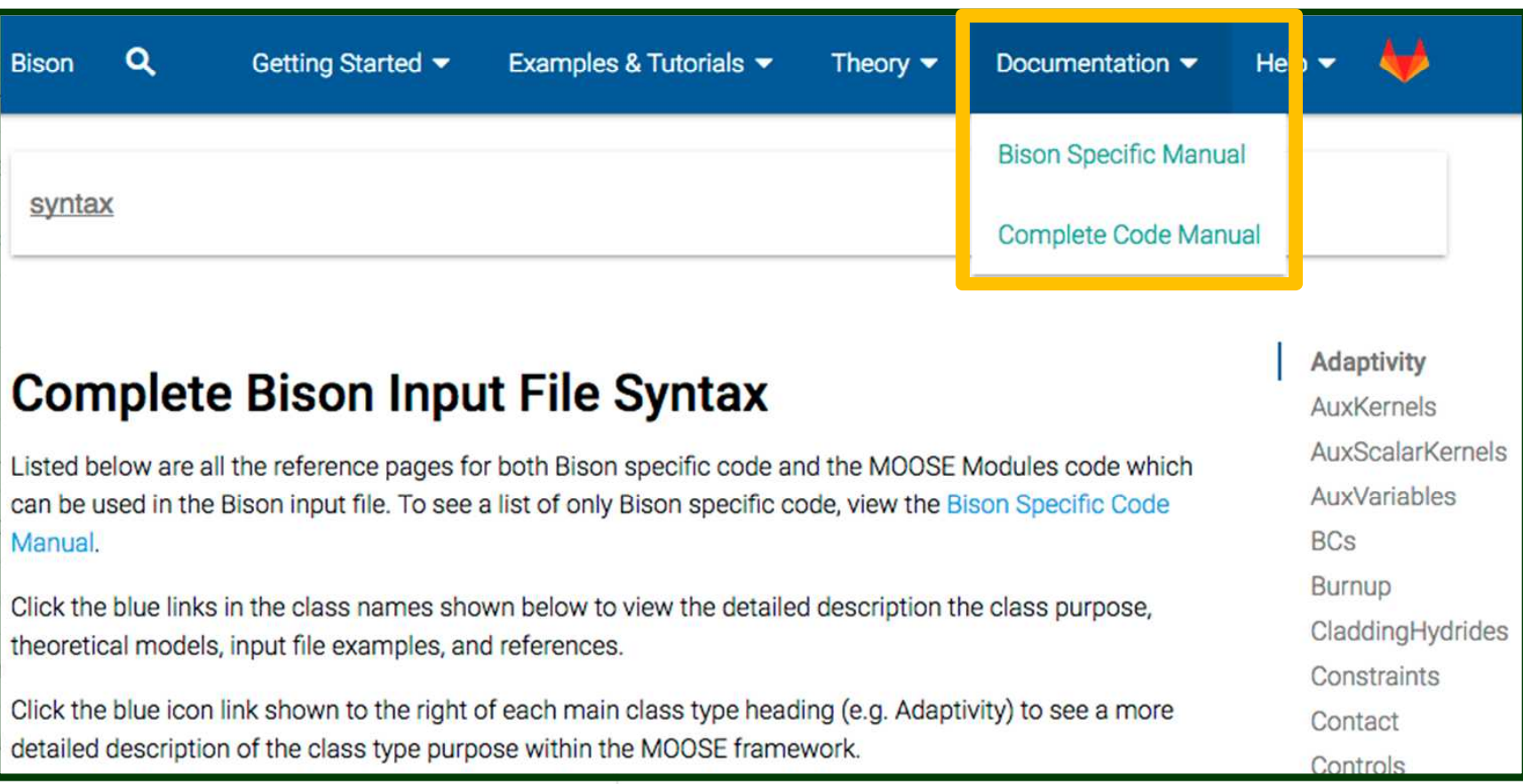

FeCrAlOxideAux Calculates the oxidation and corrosion of FeCrAl cladding 
 Embedded MOOSE Module Pages}

\section{Access} information about each possible input file block

Remain within the single unified documentation website

- Eliminates informal requirement to know which classes are Bison and which are MOOSE classes

\section{Compute Axisymmetric RZ Finite Strain}

Compute a strain increment for finite strains under axisymmetric assumptions.

\section{Description}

The material ComputeAxisymmetricRZFiniteStrain calculates the finite strain for 2D Axisymmetric systems.

Axisymmetric (cylindrical) materials are included in Tensor Mechanics for revolved geometries and assume symmetrical loading. These 'strain calculator' materials compute the strain within the appropriate coordinate system and rely on specialized AxisymmetricRZ kernels to handle the stress divergence.

\section{WARNING: Symmetry Assumed About the $z$-axis}

The axis of symmetry must lie along the $z$-axis in a $(r, z, \theta)$ or cylindrical coordinate system. This symmetry orientation is required for the calculation of the residual and of the jacobian. See StressDivergenceRZTensors for the residual equation and the germane discussion.

The AxisymmetricRZ material is appropriate for a $2 \mathrm{D}$ simulation and assumes symmetry revolved about the z-axis. A $2 \mathrm{D}$ formulation of an appropriate simulation problem can reduce the simulation run time while preserving key physics. Axisymmetric simulations are appropriate to problems in which a solid is generated by revolving a planar area about an axis in the same plane.

\section{NOTE: Use RZ Coordinate Type}

The coordinate type in the Problem block of the input file must be set to COORD_TYPE = RZ.

Axisymmetric Strain Formulation

The axisymmetric model employs the cylindrical coordinates, $r, z$, and $\theta$, where the planar cross section formed by the $r$ and $z$ axes is rotated about the axial $z$ axis, along the length of the cylinder, in the $\theta$ direction. The cylindrical coordinate system strain tensor for axisymmetric problems has the form

$$
\left[\begin{array}{ccc}
\epsilon_{r r} & \epsilon_{r z} & 0 \\
\epsilon_{z r} & \epsilon_{z z} & 0 \\
0 & 0 & \epsilon_{\theta \theta}
\end{array}\right]
$$




\section{Experienced User: New Classes \\ Require New Documentation}

Nuclear Energy

Enforce connection between documentation and source code by requiring markdown files for every class in Bison

- Merge requests will fail Civet testing without corresponding markdown files

[ /projects/bison/doc](devel)> ./moosedocs.py build --check

MooseDocs.extensions.appsyntax: Reading MOOSE application syntax.

MooseDocs.extensions.appsyntax: Building MOOSE class database.

MooseDocs.build: Cleaning destination/Users/pittsa/.local/share/moose/site

MooseDocs.commands.check: No documentation for /Materials/ComputeCrystalPlasticitystress.

- The page should be located at /Users/pittsa/projects/moose/modules/tensor_mechanics/doc/content/source/ materials/ComputeCrystalPlasticityStress.md.

- It is possible to generate stub pages using './moosedocs.py check --generate'.

Markdown files should contain theory geared toward the user

- Input parameters table and example usage blocks are automatically generated by MooseDocs

- General documentation standards are available: http://www.mooseframework.org/moose/utilities/MooseDocs/generate.html 


\section{Future Development Goals}

- To-date documentation development efforts have focused on light water reactor mechanics materials

- Complete tutorial sections with a focus on advanced fuel

- Including Metal fuel, TRISO fuel, and ATF

- Integrate training workshop materials in an interactive manner

- Migrate assessment case information to documentation website 
Nuclear Energy

\section{Bison Documentation Website}

https://hpcsc.inl.gov/ss|/BISON/site/ (Requires a Bison license, Built daily from Bison's master branch)

https://bison.inl.gov/Documentation/index.aspx (External)

With a bison-opt executable, build the documentation locally

cd $\sim /$ projects/bison/docs

./moosedocs.py build --serve 